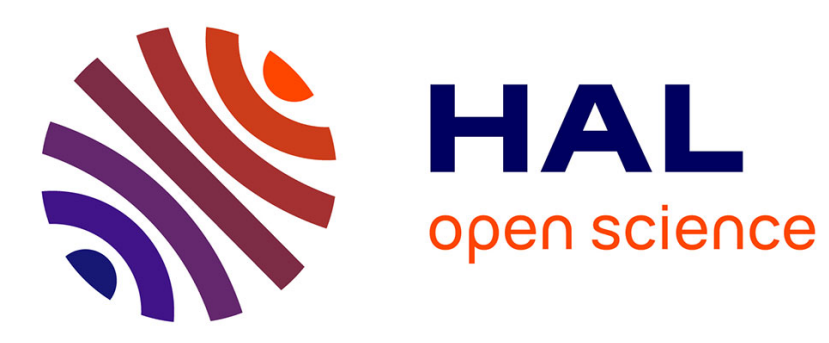

\title{
Addiction à l'alcool, objet concret et régulation des éprouvés internes
}

Guillaume Poupard, Guy Gimenez, Jean-Louis Pedinielli

\section{To cite this version:}

Guillaume Poupard, Guy Gimenez, Jean-Louis Pedinielli. Addiction à l'alcool, objet concret et régulation des éprouvés internes. Revue de psychothérapie Psychanalytique de Groupes, 2004, Le groupe et la question sexuelle, 43 (2), pp.159-173. 10.3917/rppg.043.0159 . hal-01386493

\section{HAL Id: hal-01386493 \\ https://hal-amu.archives-ouvertes.fr/hal-01386493}

Submitted on 24 Oct 2016

HAL is a multi-disciplinary open access archive for the deposit and dissemination of scientific research documents, whether they are published or not. The documents may come from teaching and research institutions in France or abroad, or from public or private research centers.
L'archive ouverte pluridisciplinaire $\mathbf{H A L}$, est destinée au dépôt et à la diffusion de documents scientifiques de niveau recherche, publiés ou non, émanant des établissements d'enseignement et de recherche français ou étrangers, des laboratoires publics ou privés. 


\title{
ADDICTION A L'ALCOOL, OBJET CONCRET ET RÉGULATION DES ÉPROUVÉS INTERNES
}

\author{
GUILLAUME POUPARD \\ GUY GIMENEZ \\ JEAN-LOUIS PEDINIELLI
}

PREAMBULE

Le terme « addiction » (ad-dicere : " dire à », au sens de attribuer quelqu'un à une autre personne) est un vieux terme français provenant du latin qui désignait en droit : la contrainte par le corps de celui qui, ne pouvant s'acquitter de sa dette, était mis à la disposition du plaignant par le juge. Aujourd'hui, «Addiction, [...] désigne la répétition d'actes susceptibles de provoquer du plaisir mais marqués par la dépendance à un objet matériel ou à une situation recherchés et consommés avec "avidité" ». (J.-L. Pédinielli et coll. 1997). Ce terme a été réintroduit et défini par Goodman en 1990 comme un processus dans lequel est réalisé un comportement pouvant procurer du plaisir ou réduire un état de souffrance intérieur, mais qui est caractérisé par l'échec répété de son contrôle et sa persistance en dépit des conséquences négatives repérées par le sujet de l'acte. Il a alors regroupé à partir de cinq critères les différentes modalités comportementales des conduites de dépendance.

Concernant plus spécifiquement l'addiction à l'alcool, de nombreuses définitions ont été dnnnées pour caractériser le, lien de

Guillaume Poupard, psychologue clinicien, chargé de cours, université de Provence/Centre de recherche Psy/CLE/g.poupard@ mageos.com.

Guy Gimenez, maître de conférences en psychologie clinique, université de Provence/Centre de recherche PsyCLElguy-gimenez2@ wanadoo.fr

Jean-Louis Pédinielli, professeur en psychologie clinique, université de Provence/Centre de recherche Psy/CLEi'Jean-Louis .Pedinielli@wanadoo.fr 
lhomme avec le produit alcool, traduisant par là même les paradigmes théoriques auxquels ces auteurs se référaient. Si la définition que proposait P. Fouquet (1951,231-251) est aujourd'hui toujours d'actualité : « II y a alcoolisme lorsqu'un individu a, en fait, perdu la liberté de s'abstenir de l'alcool », elle se trouve être précisée par l'approche étiopathogénique d'usage qui s'étaie sur une plurifactorialité et par deux approches complémentaires qui permettent de classer les conduites d'alcoolisation sur le plan nosographique. La première est le modèle bidimensionnel qui délimite la notion d'abus ou d'utilisation nocive pour la santé du syndrome de dépendance (G. Edward, 1978 ; CIM 10 1992, classification de l'OMS 1992, DSM-IV 1994). La seconde, typologique, est une description de sous-groupes d'alcoolismes possédant des caractéristiques homogènes (Knight, 1937 ; Jellinek, 1960,Tarter et coll., 1977 ; Cloninger et coll., 1978 ; Zucker, 1987 ; Babor, 1992 ; Radouco-Thomas et coll., 1979 ; Schuckit, 1985). Ainsi, en l'état actuel des recherches, l'alcoolisme est appréhendé comme un trouble bio-psychosocial dont le déterminisme est toujours plurifactoriel, ce qui nécessite une approche éclectique et intégrative. Dans le cadre de cet article, nous nous référerons plus particulièrement aux modèles psychanalytiques de l'addiction (Me. Dougall, 1978, 1982 ; Gutton, 1984 ; Jeammet, 1994,1995) ainsi qu'à certains modèles psychanalytiques de l'alcoolisme (Mijolla, Shentoub, 1973 ; Monjauze, 1991, 1999).

\section{PRESENTATION DU DISPOSITIF}

\section{Introduction}

Le dispositif thérapeutique et exploratoire que nous proposons aux patients présentant une addiction à l'alcool que nous accueillons en centre d'alcoologie clinique possède des caractéristiques que nous considérons comme étant à l'origine des informations que nous avons recueillies et présentées dans le cadre de notre recherche en psychologie clinique et pathologique (G. Poupard, 2002). Ce dispositif, nous l'avons « rêvé pour ces patients » (R. Kaës, 2001) et continuons à le rêver avec eux. Ce faisant, sa progressive mobilité est liée aux enseignements donnés par les groupes qui le traversent, mais il se façonne aussi par son inscription temporelle au fur et à mesure des aventures intersubjectives et groupales qui s'y déploient. Cette légère variabilité de dispositif, si elle n'affecte pas la rigueur des informations que nous recueillons, nécessite cependant que nous lisions les subtils déplacements qui s'y opèrent comme des éléments singuliers du travail thérapeutique à l'œuvre (dépôts transférentiels sur le dispositif). Nous suivons en cela R. Kaës $(1980,54)$ qui considère le dispositif clinique comme « l'ensemble des aménagements prédéterminés définissant les conditions constantes d'une expérience [...] soit l'aménagement du temps et de l'espace, la structure des modalités relationnelles et expressives prescrites par la règle, la détermination du nombre et du statut (le plus souvent : âge, sexe, statut professionnel) des participants ».

\section{Description du dispositif}

Les patients que nous accueillons en centre d'alcoologie clinique sont majeurs, de sexe masculin ou féminin et sont hospitalisés sur libre consentement. Ils présentent différents troubles somatiques et psychiques, liés à l'utilisation répétée et pathologique de l'alcool.

Les groupes qui font l'objet de notre étude sont des groupes à visée thérapeutique et exploratoire, construits à partir d'une adaptation du modèle du psychodrame psychanalytique de groupe et du jeu de rôle de formation personnelle. La participation à ces groupes dits « ouverts » (entrées et sorties permanentes des patients) s'inscrit dans le dispositif de soin proposé par l'institution.

Les séances ont lieu une fois par semaine pour chaque groupe (même horaire, même lieu, mais jours différents), sous la forme suivante : quarante minutes de séance groupale, vingt minutes de pause, quarante minutes de séance groupale, fin des séances hebdomadaires. Lors du temps de pause et à l'issue de la deuxième séance, les moniteurs de groupe se retrouvent dans une salle, autre que celle des séances, pour s'engager dans un travail d'élaboration de la dynamique des séances (repérage des contenus manifestes et latents, repérage des transferts et de la dynamique groupale, définition des axes stratégiques d'intervention...).

Les moniteurs de groupe sont au nombre de trois (deux, présents sur une durée de deux mois - en quinconce - et un, présent toute l'année) dans chacun des groupes. Tout changement ou toute absence d'un moniteur de groupe, ainsi que tout départ d'un des participants, est énoncée en grand groupe en fin de séance. La présence de trois moniteurs de groupe observant et intervenant dans le processus thérapeutique en train de se dérouler est à la fois entendue comme une variable produisant des effets pluriels mais aussi comme une source véritable d'information des processus à l'œuvre. Son analyse rigoureuse est susceptible d'apporter de nombreux éléments de compréhension de l'interaction groupale qui s'y déploie et du fonctionnement psychique des différents participants (analyse des processus intertransférentiels, R. Kaës, 1976,1982). Enfin, il est défini que l'absence de deux moniteurs de groupe ou la présence d'un seul patient entraîne un non-déroulement de séance.

Des règles précises de fonctionnement du groupe, propres à chacun des trois temps du dispositif (construction, jeu, élaboration), sont 
énoncées en début de chaque première séance hebdomadaire, rendant ainsi possible une réflexion personnelle (sur soi et sur ses modes de relation aux autres) et de groupe.

Des variations méthodologiques à la cure psychanalytique ont été apportées par notre dispositif groupai et méritent d'être ici mises en exergue. En effet, après P. Ricœur (1986) et R. Kaës (1994, 1998, 2002) nous nous sommes questionnés sur les formes de la réalité psychique accessibles par le recours à un dispositif groupai : «Quelle sorte de phénomènes psychiques la situation de groupe sélectionne et retient spécifiquement?»

Parmi les différentes caractéristiques du dispositif psychanalytique de groupe (la présence simultanée en face-à-face de plusieurs personnes, l'agencement des liens intersubjectifs dans un appareil de liaison et de transformation des formations psychiques, l'interdiscursivité des processus associatifs, les effets de travail psychique consécutifs à ces trois caractéristiques), nous insistons en particulier ici sur la réintroduction spécifique du rapport entre les processus associatifs verbal et non-verbal et sur linvitation à la réouverture de la place au regard qui permettent que se chevauchent le pictogrammique et la parole. Ce faisant, nous pouvons avancer que le dispositif groupai que nous proposons promeut un chevauchement - à des niveaux quantitatifs et qualitatifs différents - des actes, des gestes, des sons et des paroles lors des trois temps fondamentaux d'une séance (temps d'émergence d'un scénario, temps de jeu, temps de reprise du scénario) ; ce qui ouvre une large place aux éléments non-verbaux, éléments les plus proches des traces pictogrammiques.

Enfin, soulignons que les aménagements spécifiques de notre dispositif ont été envisagés de façon progressive, et ce, en tenant compte des résistances des différents participants mais aussi des phénomènes groupaux.

MÉTHODOLOGIE DE RECUEIL ET DE PRÉSENTATION DES DONNÉES

Notre matériel d'étude est constitué de 340 séances de groupe, soit environ 250 heures de vidéo. La décision d'utiliser une caméra vidéo comme support de recueil des données cliniques s'est accompagnée d'une réflexion critique quant aux informations que nous allions obtenir et aux conclusions auxquelles nous pouvions aboutir eu égard aux différentes caractéristiques du dispositif et du matériel vidéo (angle de prise de vue et temps de la séance ; dimension de la salle et focale de la caméra vidéo). Nous gardons alors présent à l'esprit que nos choix méthodologiques ont des conséquences sur le type de traces disponibles lors du traitement ultérieur, mais aussi sur le processus thérapeutique à l'œuvre (refus de jouer ou de parler, construction de contenants de pensée ou de schèmes d'enveloppes...). De plus, parce qu'une des limites essentielles d'une caméra vidéo est de recueillir les informations qui traversent son champ de prise de vue, nous sommes aussi attentifs aux informations « hors champ » (temps de pose, éprouvés internes et pensées non formulées...) que nous retranscrivons lors des temps de réflexion postgroupe.

De fait, nous prenons l'option de lire les réponses des sujets à ces éléments du cadre, à la fois comme des indicateurs des processus à l'œuvre au niveau intrasubjectif, intersubjectif et groupai (effet de résonance de type projectif), mais aussi comme des effets produits par les caractéristiques intrinsèques du support utilisé et de la méthode employée. Il nous semble ainsi qu'est rendu possible le chevauchement de l'aire de la réalité externe et celle des phénomènes inconscients individuels et groupaux.

Concernant notre méthodologie de présentation des données cliniques, nous utilisons après G. Gimenez (1993) un tableau à trois colonnes permettant de distinguer trois types de données cliniques :

- les données sensorielles (première colonne) qui sont les faits cli niques repérables à partir des organes des sens et en particulier les faits cliniques visuels, auditifs et olfactifs ;

- les données internes des moniteurs de groupe (deuxième colonne) qui sont toutes les associations et les éprouvés ressentis par eux, qu'ils soient nommés ou non ;

- les hypothèses sur les processus psychiques individuels ou de groupe

(troisième colonne, textes entre crochets), mais aussi les types d'inter ventions et de stratégies proposées par les moniteurs de groupe qui sont le résultat d'un travail de transformation intellectuelle eu égard à leurs référentiels théoriques.

Enfin, même si ce travail mental de transformation et de construction s'appuie sur des concepts psychanalytiques, il est important de garder présent à l'esprit le « détournement » qui est ici opéré hors de leur contexte d'origine. En effet, les données que nous recueillons sont celles qui émergent dans le cadre d'un travail thérapeutique groupai inscrit dans un protocole institutionnel de soin et non dans le cadre d'une cure analytique.

\section{AIRE DU CHERCHEUR, AIRE DU CLINICIEN}

La recherche en psychologie clinique ouvre la possibilité de laisser se chevaucher deux aires de travail pouvant répondre à des logiques différentes. Si le point de nouage entre la position du clinicien et celle du chercheur s'établit autour des faits - auxquels l'un et l'autre sont liés -, leurs objectifs divergents peuvent les conduire à adopter des positions cliniques, méthodologiques et stratégiques parfois antinomiques. Mais, peuvent aussi apparaitre deux exigences épistémologiques ne répondant pas à la même temporalité (les échéances définies 
dans le cadre d'une recherche ne sont pas nécessairement les mêmes que celles d'une rencontre clinique). De cette conflictualité entre deux aires singulières, ainsi soutenues, peuvent émerger des éléments de connaissance inscrits dans une praxis.

\section{HYPOTHESE}

Notre hypothèse de travail est que le recours possible à des objets concrets dans un dispositif thérapeutique groupai, utilisant la mise en jeu de scénarisations psychodramatiques, permet à des patients présentant une addiction à l'alcool de faire l'expérience in situ d'une meilleure régulation de leurs éprouvés internes et d'intégrer à long terme cette capacité de régulation.

Nos critères de mise à l'épreuve de notre hypothèse permettent de repérer trois temps consécutifs dans une séance groupale. Nous pouvons tout d'abord constater l'état de tension interne et la non-régulation de celui-ci par les patients. Puis, le recours à un objet concret comme un facteur essentiel de régulation émotionnelle. Enfin, la modification de l'état émotionnel initial des patients et l'intégration du processus transformationnel.

\section{SITUATIONS CLINIQUES}

\section{«Le groupe du parfum » ou l'étayage sensoriel sur un objet concret}

\section{L'état de tension interne ou l'absence de mots pour le dire}

La séance du « groupe du parfum » débute par l'alternance dans la chaîne associative groupale de quelques ponctuations verbales soulignant l'absence d'idées chez les participants mais aussi par des temps plus ou moins longs de silence profond. Une des monitrices du groupe invite alors les patients à tenter de définir le processus psychique groupai à l'œuvre dans ce moment de la séance. L'état de tension interne, éprouvé par chaque participant, continue de se manifester dans ce silence contagieux dans lequel aucune pensée ne semble pouvoir émerger. L'absence de formes pour figurer l'innommable (W.R. Bion, 1962) laisse chacun des membres du groupe dans l'espace désertique de la solitude. Cependant, et après qu'a été explicitement nommée l'existence d'un indicible par un des moniteurs du groupe, M. A relie la présence du silence à ce qui n'est pas dit ou tu : «Le silence, les nondits ou les choses cachées... » Par cette intervention, il souligne à la fois la présence de pensées immobilisées ou inaccessibles et ouvre aussi et surtout les pensées du groupe à un au-delà des mots.
Etayage sur un objet concret partageable ou l'apparition des mots pour le dire

M. P intervient ensuite pour préciser où « flottent ses pensées » dans la salle : « je sens une odeur de parfum et j'essaie de deviner... »; ce qui amène alors chacun des participants du groupe à se saisir de cette « invitation au voyage olfactif » et à s'engager dans le dévoilement de cette énigme. À l'affût de l'odeur, chacun se rapproche de l'autre dans une tentative de rencontre de «l'intimité olfactive » de ses voisins. L'enveloppe groupale, c'est-à-dire « Ce réseau, qui enserre les pensées, les paroles, les actions, [et] permet au groupe de se constituer un espace interne et une temporalité propre » (D. Anzieu, 1975,1), se tisse ainsi dans cette proximité physique et psychique autour de cet objet fugace, mystérieux et invisible qui circule entre les uns et les autres membres du groupe. Cette attention particulière au sensoriel qui se déploie dans le groupe donne lieu à la construction d'un jeu autour de la thématique du parfum. Mais ceci se produira après qu'une des participantes a désigné un des moniteurs de groupe comme le propriétaire et le dépositaire de cette fragrance indéfinissable et insaisissable.

L'étayage sur les caractéristiques sensorielles de cet objet externe, expérienciables dans ce bref moment groupai, permet ainsi que soient figurées des sensations jusqu'alors suspendues et qu'adviennent, puis se prononcent des pensées impensables ou interdites à penser. Nous soulignons ici que le processus de figuration se déploie à partir de caractéristiques sensorielles d'objets concrets présents dans le dispositif et qui vont émerger dans le cadre d'une rencontre permettant aux psychés en présence de s'appareiller autour de cet objet partageable. Ainsi, nous rejoignons les travaux de recherche du COR. autour de l'objet de relation qui « doit être un objet qui puisse être repéré séparément par les deux personnes en présence et déclencher en chacun un travail de pensée » (M. Thaon, 1988,15). Objet concret externe, il appartient au champ de l'intermédiaire défini par G. Roheim (1943), D.-W. Winnicott $(1951)$, R. Kaës $(1983,1985)$ et permet notamment la mise en contact de plusieurs psychés (fonction d'interface).

Le jeu qui s'élabore s'appuie sur des éléments de réalité vécus par M. P lors de son week-end thérapeutique. Se rendant dans une parfumerie « juste pour aller sentir », il échange avec la vendeuse sur la fragrance des produits qu'elle lui présente et l'informe qu'il n'achètera rien car il est en cure d'alcoologie. Cette brève remarque restitue pour nous le rapprochement entre les représentations concernant les parfums et celles de l'alcool. Ceci prolonge la chaîne associative groupale qui se déploie ensuite mettant en exergue le chevauchement entre le plaisir et l'interdit : « plaisir pris à sentir », «pour le plaisir, [...] pas sur les mains. » Dès lors nous faisons l'hypothèse que la transgression dont M. P pense être l'auteur s'appuie sur l'émergence en lui, durant 
son week-end thérapeutique, de pensées et de désirs d'alcool. La « consommation olfactive » de parfums pouvant alors être pensée comme une « forme de déplacement agi » de son désir d'alcool. L'appareillage des psychés à partir d'un objet concret possédant des caractéristiques sensorielles spécifiques (le parfum) rend alors possible la reprise psychique des pensées suspendues, et des impensés (G. Gimenez, 2000). Par ce «passage par l'objet» (C. Guérin, 1991,123) s'initie ainsi le travail de figuration de pensées impensables. Ce faisant, nous avançons que la fragrance du parfum présente dans la salle, en ce début de séance, a permis à $\mathrm{M}$. $\mathrm{P}$ d'être en contact avec certaines pensées inaccessibles et certains affects suspendus. Ce premier temps de la séance, ouvert sur cette expérience sensorielle groupale articulée autour d'un objet concret, nous le pensons comme un élément central à la construction de la chaîne associative groupale à venir et à la modification de l'état émotionnel des participants du groupe.

\section{Étayage sur un objet concret et scénarisation psychodramatique}

M.P, «porte-sens » du groupe (R. Kaës, 1994, 231-260) nous invite ensuite à nous évader avec lui vers les marchés du Sud, burs odeurs d'épices et de denrées alimentaires. La centration sur les flaveurs évoquées initie le déploiement d'images mentales. Cependant, le processus d'invitation à la rêverie s'interrompt dès qu'il précise qu'il lui est impossible d'aller jouer une situation fictive : «Je sais pas jouer, le fictif, je sais pas le faire. Parler oui, mais je sais pas le... » Lorsqu'un des moniteurs du groupe souligne aux différents participants qu'ils disposent de tous les éléments nécessaires à l'exploration d'un jeu, M. P intervient alors à nouveau pour remarquer que si le jeu de la parfumerie peut être proposé, l'explorer en l'absence de l'objet réel lui semble impossible : -M.P: « Le parfum, c'est difficile car sans le sentir c'est... »

- Moniteur 1 : «C'est difficile, sans l'objet, sans l'odeur réelle ?» -M.P: « J'ai pas l'odeur, j'ai pas... en haut, dans la pharmacie, j'en ai ! »

- Moniteur 1: «C Ca ne serait pas du jeu.»

- M. P : « Si, ça serait du jeu... et y'aurait de la matière dedans. » -Moniteur 1 : «Est-ce qu'il n'est pas possible d'imaginer les effluves ? »

Dans cette proximité du jeu et de la réalité, l'objet concret est pensé comme pouvant permettre l'investissement de l'espace du « comme-si ». La fragrance mystérieuse sur laquelle chacun s'est appuyé, pour que se forment ses pensées et que se construise l'enveloppe groupale, est envisagée là encore comme ce qui permettrait que des pensées soient mises en représentation scénarisée puis figurées dans un jeu.
M. P acceptera cependant de jouer un client qui en conseille un autre, ce dernier venant acheter un parfum pour sa femme. L'échange entre les clients, pendant le jeu, se poursuivra dans l'espace de parole. Le prix du parfum en est l'axe central et les ressentis sensoriels et émotionnels qui semblent oubliés ou inaccessibles ne peuvent faire leur entrée dans le champ de la parole énoncée : «Comment j'ai vécu ce que j'ai ressenti ?... Je sais pas. »

\section{Métareprésentation, étayage sur des objets concrets, et régulation des éprouvés internes}

Cette séquence clinique groupale met en relief une demande récurrente des patients participant à ces séances de psychodrame : pouvoir utiliser un objet concret dans leurs mises en jeu psychodramatiques, « Pour que je joue une scène de conflit, amenez-moi mon mari », «Pour jouer le mec qui boit dans le bar, il me faut un verre avec de l'alcool. » Cependant, alors que leurs discours semblent souligner des défaillances de leurs capacités d'abstraction, nos observations cliniques nous obligent aujourd'hui à constater qu'ils peuvent jouer des scénarios sans utiliser d'objets concrets s'ils s'étayent sur des éléments de la réalité externe. Une suffisante proximité du jeu et de la réalité semblerait ainsi être une des conditions nécessaires pour que ces patients construisent et investissent dans le jeu leurs scénarisations psychodramatiques. Sans cela, celui-ci est perçu comme factice et sans intérêt du fait de son écart avec la réalité : «Le jeu n'est qu'hypocrisie », «C'est pas vrai. » Nous avançons que ce rapport spécifique que ces patients tissent entre le jeu et la réalité leur permet d'explorer à distance certains éprouvés internes issus de la rencontre intersubjective et d'éviter de ce fait de se trouver submergés par ceux-ci. Quant à l'objet concret (le parfum), il devient alors un support possible d'une régulation émotionnelle (fonction pare-excitative) et peut être pensé comme un objet de relation pour autant qu'il déclenche un travail de pensée chez les différents interlocuteurs et « représente l'état de la relation à un moment donné de la rencontre » (M. Thaon, 1988, 16 ; C. Guérin, 1992,121). Ce faisant, la fugacité, la volatilité et l'insaisissabilité partielle de la fragrance du parfum d'un des moniteurs du groupe peuvent être lues comme la forme trouvée et investie par ces patients pour soutenir les effets de la rencontre. L'objet est alors envisagé comme «le produit d'un travail psychique préalable d'une [ou plusieurs] personne qui associe une souffrance ressentie dans la relation à une forme investie au préalable par lui [ou par eux] et qui est offerte [aux autres] comme conteneur potentiel des émotions » (M. Thaon, 1988,17). 


\section{«Le groupe de la mouche» ou l'objet de dépôt des pensées du groupe}

État de tension interne ou l'expérience du «trou » dans les pensées

«Le groupe de la mouche » débute par un moment d'arrêt soudain $\mathrm{du}$ discours de la monitrice de groupe lorsqu'elle énonce les règles de fonctionnement : «J'ai un trou ! (Celle-ci évoquera lors du temps de pose qu'elle était parasitée par des événements personnels et « n'était pas là dans la séance. ») Les associations d'idées qui circulent dans le groupe en ce début de séance soulignent la difficulté de penser et de verbaliser certaines pensées. Certains évoquent même le paradoxe que produit chez eux l'énoncé de la consigne : «On dit les idées comme elles viennent. »C'est comme si penser devenait impossible, « comme si tout d'un coup on nous demandait de penser, d'habitude tout le monde pense... Mais là, comme si on s'arrêtait sur quelque chose. On pense, c'est comme ça!» Penser devient alors difficile, voire impossible. Un questionnement qui s'engage entre quelques participants du groupe autour des pensées souligne le rapport de chacun aux siennes et ce en fonction de certaines situations : «Les pensées qui font du bien, les pensées qui font du mal », «Les pensées qui font du bien et du mal, comme le décès, ça fait mal et cependant ça soulage car la personne souffrait », « Ce qui est mauvais pour l'un n'est pas forcément mauvais pour l'autre. » Puis, un participant intervient pour questionner l'interêt de ce travail groupai et de l'utilisation que les moniteurs du groupe font des pensées des participants : «Si vous voulez nos idées, c'est pour en faire quoi, pourquoi pas vos idées à vous ? » Un moment de silence envahit ensuite le groupe jusqu'à l'appel aux pensées du groupe qui est alors envisagé comme ce qui induirait un "déblocage »: «Si plusieurs donnent des idées, on pourrait s'appuyer dessus et alors on pourrait penser. » Un des patients qui évoque l'idée d'un « embouteillage » initie alors le déploiement d'images que les uns et les autres énoncent; l'image d'un « péage avec des voitures qui attendent » est évoquée, « II y a du bruit derrière et devant rien qui se passe. » Un autre participant parle ensuite d'une machine à laver et de « chaussettes noires que l'on enlève de ses pieds et il reste des petits trucs noirs sur les pieds ». Par ces pensées qui sont livrées dans le groupe et au groupe, les images qui se partagent se mêlent aux bruits et aux odeurs.

Les éléments impensés qui ont été déposés dans ce moment initial de rencontre groupale ont été condensés et mis en forme dans le moment de paralysie éprouvé et verbalisé par une des monitrices du groupe et partagé par les différents participants. L'état de tension interne initié par cette rencontre groupale souligne avec acuité la douleur de l'altérité et l'impossibilité, à ce moment-là du groupe, pour chacun des participants de s'engager dans un travail psychique de transformation et de mise en sens. Il faudra attendre l'investissement du groupe comme bon objet (M. Klein, 1934) et comme appareil à penser les pensées (W.R. Bion, 1962), pour que se déploie la chaîne associative groupale jusqu'alors mise en suspens.

\section{Etayage sur un objet concret et scénarisatlon psychodramatique}

M. A, qui vient d'arriver en retard, s'engage dans la discussion et précise qu'il pense à son week-end thérapeutique lors duquel il va aller à un concert de Serge Lama. M. B, anglais, demande à ce dernier quels sont les titres des chansons de cet auteur. Chacun se trouve alors invité à laisser résonner en lui les chansons de Serge Lama, ce que certains prolongent à haute voix. Puis, le silence s'installe à nouveau après ce moment de rencontre groupale autour d'une expérience sonore partagée. Un des moniteurs du groupe qui intervient alors en remarquant que «nous avons maintenant Serge Lama et le noir dans les pieds, au milieu » accompagne ses paroles d'un geste de la main vers le centre de l'espace formé par les chaises (le trou central). Les regards des différents participants qui suivent la direction indiquée par ses mains, découvrent, au milieu du tapis, une mouche en train de se déplacer ; chacun s'engage alors dans un mouvement d'expression libre de ses pensées :

- « Et la mouche, elle est là toute seule. »

- « Non, elle est avec Lama et les pieds. »

- «La mouche sentait les pieds noirs. »

Cet échange mené tambour battant se poursuit autour de la vie, du fonctionnement interne, de l'histoire et de la présence de ce participant ailé au centre du groupe :

«Est-ce que quelqu'un s'est intéressé à la psychologie des mouches ? Faut peut-être qu'un chercheur écrive quelque chose là-des sus. »

- «Les mouches sont pas intéressantes alors que les abeilles le sont: les mouches à miel. »

- « Nous, on observe la mouche, elle peut-être nous observe. »

- «On connaît pas la vie des mouches, les mouches n'ont pas l'air organisées, c'est chacun pour soi. »

- «Est-ce qu'elles ont un cerveau, les mouches, est-ce qu'elles pen sent, à quoi elles peuvent penser ?»

- «La mouche est peut-être la réincarnation d'un de nos ancêtres ? »

Puis, un des participants remarque intrigué que la mouche ne s'est pas envolée depuis qu'on l'observe ; ce à quoi il prête un sens tout à fait singulier : "Elle reste toujours au même endroit. Peut-être qu'elle a trop bu. Elle n'arrive pas à décoller. »Un autre participant se lève alors et tente de la pousser d'un mouvement de main, ajoutant : « Si on 
pense à elle, peut-être qu'elle va aller mieux ? » Jusqu'à la fin de cette séance les patients restent centrés autour de cette mouche, laissant leurs pensées vagabonder à partir des « faits et gestes » de cette compagne clandestine.

La mise enjeu d'une scénarisation n'advient pas dans cette séance de groupe. Chacun des patients étaye son activité associative sur ce visiteur imprévu bien qu'il reste sous l'emprise fascinante des mouvements de celui-ci. Si ce collage à un objet concret ne permet pas qu'émergé une proposition de scénario, il apparaît cependant que celui-ci engage chacun des participants du groupe dans un processus de dépôt de ses pensées à l'égard des participants du dispositif groupai et en particulier des moniteurs de groupe. Et l'on peut avancer l'hypothèse que l'absence mnésique et psychique d'une des monitrices du groupe, formulée par : «J'ai un trou », a « gelé » le processus potentiel de mise en scénarisation, par l'expérience psychique traumatogène partagée qui est venue s'inscrire en chacun des participants de ce groupe. Les saccades dans le processus associatif groupai ont témoigné de cette impossibilité à soutenir durablement les fonctions du penser (D. Anzieu, 1994) et que se réactivent les processus préconscients. $\mathrm{Ni}$ l'équivocité du signifiant embouteillage, ni les paroles chantées de l'œuvre de Serge Lama n'ont permis la construction d'une scénarisation psychodramatique. Chacun, immobile et immobilisé, s'est vu dans cette mouche qui n'arrivait pas à décoller faute de «pensées pensées ».

\section{CONCLUSION}

Nous appuyant principalement sur les travaux du paradigme psychanalytique et en particulier sur ceux traitant du psychodrame de groupe, nous avons souhaité mettre en exergue la place centrale que prend - et que nous réservons - à la sensorialité, à côté de celle de la parole et du corps, dans la prise en charge psychothérapique de patients présentant une addiction à l'alcool.

Nous nous sommes ici centrés sur une des conditions pouvant permettre l'émergence de certaines formes de la réalité psychique ainsi que le développement ou la reprise du travail de symbolisation de certaines expériences sensori-affectives.

Ainsi, à partir de notre hypothèse clinique nous en sommes venus à mettre en relief le rôle des objets concrets - dans un dispositif thérapeutique groupai utilisant la mise en jeu de scénarisations psychodramatiques - dans la régulation des éprouvés internes de patients addictifs à l'alcool.

Nous avançons aujourd'hui que l'étayage de leurs pensées sur des caractéristiques sensorielles d'objets concrets - présents en séance et ayant une forte contiguïté avec ceux de la réalité - leur permet de construire et d'investir dans le jeu des scénarisations psychodrama- tiques ainsi que de s'engager dans un processus transformationnel de type « régulation des éprouvés internes ». En effet, au regard de la définition métapsychologique des conduites d'alcoolisation de patients addictifs à l'alcool que nous avons précédemment proposée et qui nous avait conduit à mettre en relief l'existence d'une défaillance fonctionnelle de l'activité de liaison du préconscient (G. Poupard, 2002), nous soutenons que la fonction de figuration des objets concrets permet que se déploie et se soutienne le processus de symbolisation, c'est-à-dire que s'atténue la dysliaison entre les représentations de choses, les représentations de mots et les affects associés. Cette dernière ayant pour conséquence majeure de laisser certaines traces d'expériences sensori-affectives dans un état qualifié de «brut » (non symbolisé).

Cependant, si les séances groupales de jeu psychodramatique ont mis en exergue l'intérêt dans le travail clinique de l'utilisation de supports sensoriels concrets ainsi que l'insistance de ces patients à s'appuyer sur ces derniers dans leurs productions scéniques, nous continuons à nous questionner quant à leur caractère d'absolue nécessité. En effet, nous avons remarqué que l'utilisation d'objets de la situation vécue ou d'objets de forte proximité avec celle-ci, pouvait faciliter le jeu et le travail psychique (notamment en permettant de ne pas sombrer dans « l'ivresse de l'imaginaire », que se délie le processus de libre association et que fléchisse le seuil d'angoisse), mais n'apparaissait pas dans chacune des séances groupales. Ceci nous a amené à mettre en lumière d'autres supports d'étayage et en particulier certaines caractéristiques psychiques des cliniciens (G. Poupard, 2002).

À l'issue de ce travail d'élaboration clinique, nous réaffirmons que ces objets, par leur contiguïté avec ceux de la situation réelle, permettent l'émergence ou la reprise du processus de penser et ce à partir du moment où les patients peuvent s'étayer sur leurs propriétés sensorielles. Nous qualifions ces objets concrets de la dénomination « d'initiateurs de pensée ».

\section{BIBLIOGRAPHIE}

ANZIEU, D. 1975. Le groupe et l'inconscient. L'imaginaire groupai, Paris, Dunod.

ANZIEU, D. 1994. Le penser. Du Moi-peau au Moi-pensant, Paris, Dunod. BION, W.R. 1962. "Une théorie de l'activité de pensée » Réflexion faite, Paris, Presses universitaires de France, 1983,125-135.

CLONINGER, C.R. ; CHRITIANSEN, K.U. ; REICH,!. et coll. 1978. " Implications of sex différences in thé prevalence of antisocial personality, alcoholism, and criminality for familial transmission », Arch. Gen. Psych., 35, 941-950.

COSNIER, J. 1991. « Du côté du non-verbal », Revue de psychothérapie psychanalytique de groupe, 17, 97-99, Toulouse, ères. 
EDWARD, G. ; GROSS, M.-M. ; Keller, M. ; MOSER, J. ; ROOM, R. 1978. «Incapacités liées à la consommation d'alcool », Publications OMS, 32 Genève.

FOUQUET, P. 1951. "Réflexions cliniques et thérapeutiques sur l'alcoolisme », Évolution psychiatrique, 11, 231 -251.

GIMENEZ, G. 1993. Les hallucinations psychotiques, thèse de doctorat de psychologie clinique, Aix-en-Provence.

GOODMAN, À. 1990. «Addiction : définition and implications », British Journal of Addiction, 85, 1403-1408.

GUELFI, J.-D. ; BOYER, P. ; CONSOLI, S. ; OLIVIER-MARTIN, R. 1987. Psychiatrie, Paris, PUF.

GUERIN, C. 1991. "Chaîne associative groupale et objet de relation », dans Revue française de psychothérapie psychanalytique de groupe, $\mathrm{n}^{\circ} 17$, 123-132, Toulouse, ères.

GUERIN, C. 1992. «L'objet de relation ou la transparence de l'obstacle, à propos du film de W. Wenders, Paris Texas », Actes des journées d'études du COR. Arles, hôpital Joseph-Imbert, 117-147.

GUTTON, P. 1984. «Pratiques de l'incorporation », Adolescences, 2,315-338.

JEAMMET, P. 1994. «Dépendance et séparation à l'adolescence, point de vue psychodynamique », dans D. Bailly, J.-L. Venisse (sous la direction de) Dépendance et conduites de dépendance, Paris, Masson.

JEAMMET, P. 1995. «Psychopathologie des conduites de dépendance et d'addiction », Cliniques méditerranéennes, 47/48,155-175.

JELLINEK, E.M. 1960. «Alcoholism : genus and some of its species », Can. Med.Assoc. J., 83, 1341-1345.

KAËS, R. 1976. "Analyse intertransférentielle, fonction alpha et groupe conteneur », L'évolution psychiatrique, 1, 339-347.

KAËS, R. 1980. L'idéologie : études psychanalytiques, Paris, Dunod-Bordas.

KAËS, R. 1982. «L'intertransfert et l'interprétation dans le travail psychanalytique groupai », dans R. Kaës, A. Missenard et coll. (sous la direction de) Le travail psychanalytique dans les groupes, $H$, les voies de l'élaboration, Paris, Dunod.

KAËS, R. 1983. "La catégorie de l'intermédiaire et l'articulation psychosociale », Bulletin de psychologie, 26, $\mathrm{n}^{\circ} 360,587-593$.

KAËS, R. 1985. "La catégorie de l'intermédiaire chez Freud : un concept pour la psychanalyse ? », L'évolution psychiatrique, 50-4.

KAËS, R. 1994. La parole et le lien, Paris, Dunod.

KAËS, R. 1994. «Une fonction phorique. Le porte-parole », dans La parole et le lien, 231-260, Paris, Dunod.

KAËS.R. 1998. «Le jeu psychodramatique : modèle de figurabilité et travail du préconscient », Revue de psychothérapie psychanalytique de groupe, 30,93-106, Toulouse, ères.

KAËS, R. 2001. Intervention lors du séminaire théorique et clinique, Paris, CEFFRAP.
KAËS, R. 2002. « La consistance psychanalytique de la psychothérapie psychanalytique de groupe », dans F. Richard et coll., Le travail mental du psychanalyste en psychothérapie, Paris, Dunod.

KLEIN, M. 1934. «Contribution à l'étude de la psychogenèse des états maniacodépressifs », Essais de psychanalyse, Paris, Payot, 1968, 311-340.

KNIGHT, RP. 1937. «Dynamics and treatment of chronic alcohol addiction », Bull. Menninger Clin., 1, 233-250.

Me DOUGALL, J. 1978. Plaidoyer pour une certaine anormalité, Paris, Gallimard.

Me DOUGALL, J. 1982. Théâtre du Je, Paris, Gallimard.

MiJOlla, A. ; SHENTOUB, S.-A. 1973. Pour une psychanalyse de l'alcoolisme, 1981, Paris, Payot.

MONJAUZE, M. 1991. La problématique alcoolique, Paris, Dunod.

MONJAUZE, M. 1999. La part alcoolique du Soi, Paris, Dunod.

PEDINIELli, J-.L. ; ROUAN, G. ; BERTAGNE, P. 1997. Psychopathologie des addictions, Paris, PUF.

POUPARD, G. 2002. Addiction à l'alcool, dispositif psychothérapique et régulation des éprouvés internes, DEA de psychologie clinique et pathologique, sous la direction de J.L. Pedinielli, G. Gimenez, Aix-en-Provence.

RADOUCO-THOMAS, S. ; GARCIN, F. ; LACROIX, D. et coll., 1979. « Major concepts and trends in alcoholism », dans Alcoholism, a Multidisciplanary Approach, J. Mendlewics, H. Van Praag, Bàsel, Karger (eds).

RICOEUR, P. 1986. « La psychanalyse confrontée à l'épistémologie », Psychiatrie française, $\mathrm{n}^{\circ}$ spécial, 11-23.

ROHEIM, G. 1943. Origine et fonction de la culture, Paris, Gallimard, 1972.

SCHUCKIT, M.A. 1985. "Thé clinical implications of primary diagnostic group among alcoholics », Am. J. Psych., 143, 140-147.

TARTER, R.E. ; Me BRIDE, H. ; BUONPANE, N. et coll., 1977, « Différenciation of alcoholics », Arch. Gen. Psych., 34,761-768.

THAON, M. 1988. «Caractéristiques et fonctions des objets de relation », dans Après Winnicott. La place de l'objet dans le travail clinique, Actes des journées d'études du COR, Arles, hôpital Joseph-Imbert, 13-17.

WINNICOTT, D.-W. 1951. «Objets transitionnels et phénomènes transitionnels », Jeu et réalité, Paris, Gallimard, 7-39.

ZUCKER, R.A. 1987. «Thé four alcoholism : a developmental account of thé etiologic process », Alcohol and addiction behaviour, PC River (ed), Lincoln, Nebraska, University of Lincoln Nebraska Press, 27-83. 